\title{
Reproductive plasticity in the reef coral Echinopora lamellosa
}

\author{
Tung-Yung Fan ${ }^{1,2}$, Chang-Feng Dai ${ }^{1, *}$ \\ 'Institute of Oceanography, National Taiwan University, Taipei, Taiwan, ROC \\ ${ }^{2}$ National Museum of Biology and Aquarium, 2 Houwan Road, Checheng, Pingtung 944, Taiwan, ROC
}

\begin{abstract}
The reproductive timing and mature egg size of the reef coral Echinopora lamellosa were different between the northern Taiwan and southern Taiwan populations. A reciprocal transplant experiment was conducted to study the influences of environment on the observed differences in reproductive traits between the 2 populations. The reproductive timing of the transplanted colonies in southern Taiwan and mature egg size of the transplanted colonies in both northern and southern Taiwan changed, compared to those of the original populations. This suggests that these reproductive traits are plastic and environmental factors may play an important role in regulating them. Furthermore, the growth of early stage oocytes was faster and the spawning began 2 mo earlier in the transplanted southern Talwan colonies than those of the original populations in northern Taiwan. This suggests that the timing of reproductive activity of the transplanted E. lamellosa in southern Taiwan may be accelerated by the warmer seawater temperature.
\end{abstract}

KEY WORDS: Reproductive timing - Egg size - Phenotypic plasticity - Reciprocal transplant experiment - Reef coral

Distinguishing between phenotypic plasticity induced by proximate environmental variation and genetic divergence is crucial to understanding the ecological and evolutionary significance of life history variation (Stearns 1992). Variations in reproductive traits have been documented among geographic populations of coral species (Harrison \& Wallace 1990, Richmond \& Hunter 1990). However, the relative contribution of genetic and environmental sources to reproductive variation is rarely studied.

The reproductive timing of corals has been related to various environmental factors (Harrison \& Wallace 1990). Some studies suggest that seasonal variation in sea temperature may be the major proximate environmental factor controlling the annual cycle of reproduc-

-Corresponding author. E-mail: corallab@ccms.ntu.edu.tw tion (Babcock et al. 1986), while others indicate that sea temperature may not operate as a primary factor controlling the seasonality of reproduction (Babcock et al. 1994, Fan \& Dai 1995, 1998). As correlative evidence is conflicting and does not prove causality, rigorous experimental studies to prove the relationships between environmental factors and reproductive timing are needed (Harrison \& Wallace 1990).

Reciprocal transplant experiments have been used to illustrate the morphological plasticity (Foster 1979, Willis 1985, Bruno \& Edmunds 1997) and physiological adaptation to light (Falkowski \& Dubinsky 1981) in corals. This method offers an approach to evaluate whether or not the observed variations represent phenotypic plasticity.

The reef coral Echinopora lamellosa is a hermaphroditic broadcast spawner with external fertilization and larval development (Willis et al. 1985, Babcock et al. 1986). In a previous study, we found that gametogenesis of the northern Taiwan (NT) population of $E$. lamellosa started earlier and spawning occurred earlier or in the same month, as the southern Taiwan (ST) population (Fan \& Dai 1995). In addition, the mature egg size of the NT population was larger than that of the ST population. In this study, we used a reciprocal transplant experiment to investigate the influences of environment on the observed differences in reproductive traits between the 2 spatially separated populations.

Materials and methods. Study site: Our study sites

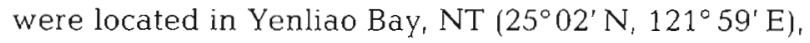
and Nanwan Bay, ST (21 $\left.55^{\prime} \mathrm{N}, 120^{\circ} 45^{\prime} \mathrm{E}\right)$, where the sexual reproduction of Echinopora lamellosa has been studied previously (Fan \& Dai 1995). Monthly mean sea temperatures range from 18.0 to $28.0^{\circ} \mathrm{C}$ and 23.5 to $28.0^{\circ} \mathrm{C}$, water turbidity ranges from 0.65 to $2.30 \mathrm{NTU}$ and 0.28 to 1.90 NTU in Yenliao Bay, NT, and Nanwan Bay, ST, respectively (Fan \& Dai 1995). 
Transplantation: In August 1992, 72 whole or partial colonies of Echinopora lamellosa in NT were removed using hammer and chisel, placed in several large buckets, immersed in seawater, and transported by car to ST within $12 \mathrm{~h}$. Substrata were cleared and the next day colonies were cemented to the reef surface along three $10 \mathrm{~m}$ transects. Fifty colonies of E. lamellosa in ST were transported to NT by the same method. However, most of the colonies were lost prior to being cemented when the buckets were swept away by strong currents. The remaining 5 colonies were cemented along one $10 \mathrm{~m}$ transect in NT in September 1992. The position of the transplanted colonies relative to the transects and other corals was mapped for later survey and collection of tissue samples. Area of the transplanted colonies, measured for 2 mo after transplantation, ranged from 17.1 to $71.8 \mathrm{~cm}^{2}$ and 19.8 to $806.4 \mathrm{~cm}^{2}$ in NT and ST, respectively.
Sexual reproduction: After 20 and 33 mo of transplantation in ST and NT, we started to examine the reproduction of the Echinopora lamellosa colonies. The examination of gametogenesis started 1 yr later in NT, when the transplanted colonies were large enough for sampling. Coral samples of 2 to 10 healthy (with normal coloration and polyp activities) and large transplanted colonies ( $>85 \mathrm{~cm}^{2}$ ) and 10 large native colonies (>314 $\mathrm{cm}^{2}$ ) were collected for reproductive studies. In order to reduce sampling damage, not all of the same colonies, either the transplanted or native colonies, were sampled repeatedly in each month. Histological preparation of samples, oocyte measurement, and the classification of developmental stages were conducted according to the procedures used by Fan \& Dai (1995). The length and width of at least 6 oocytes in nucleolar section were measured to represent the diameter of oocytes for each colony. The 5 developmental stages

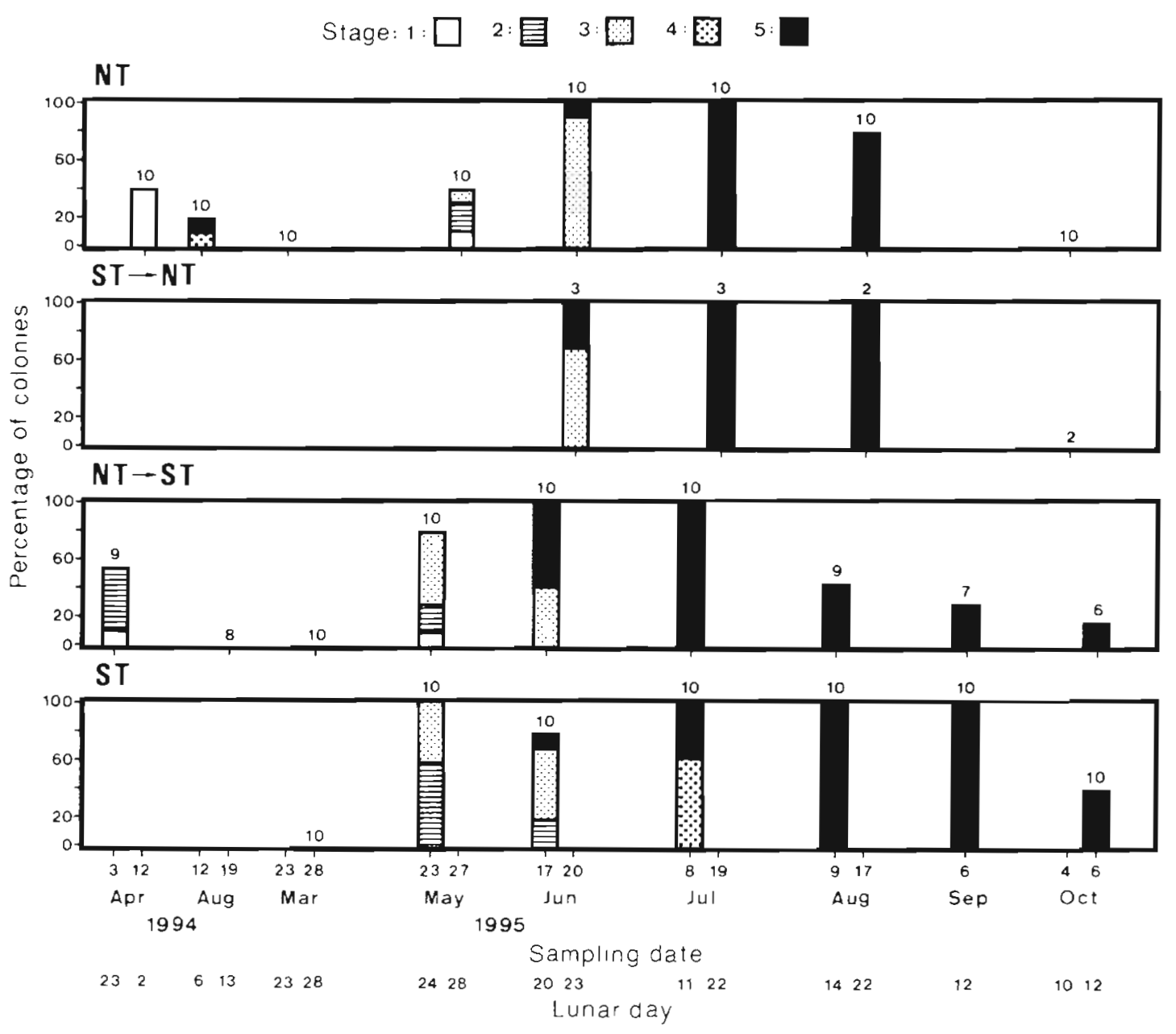

Fig. 1. Echinopora lamellosa. Changes in percentage of the native and transplanted colonies containing gametes in northern Taiwan (NT) and southern Tawan (5T). Numbers above the bars indicate the number of colonies sampled. The 5 developmental stages of gametes are (1) oogonia, (2) developing oocytes, (3) developing oocytes and early spermaries, (4) developing oocytes and spermaries, and (5) mature oocytes and spermaries. Full moon is Lunar day 15 
of gametes are (1) oogonia, (2) developing oocytes, (3) developing oocytes and early spermaries, (4) developing oocytes and spermaries, and (5) mature oocytes and spermaries. Variation in mean oocyte diameter and frequency of colonies containing different stages of gametes were used to determine the patterns of gametogenesis and spawning

Data analyses: For the comparison of oocyte diameters between populations, 1-way ANOVA was used to detect differences. A 2-way ANOVA was used to analyze the mature egg size of the transplanted and native colonies in NT and ST.

Results. Survival rate: Fifty-three (74\%) and $3(60 \%)$ of the transplanted colonies survived in ST and NT when we started to examine their reproduction in April 1994 and June 1995, respectively.

Reproductive seasonality: The month of spawning was inferred from the disappearance of mature gametes between samples from 2 consecutive months. In addition, the spawning date was presumed to be Lunar day 21, based on the finding that Echinopora lamellosa spawned on $6 \mathrm{~d}$ after a full moon in the Great Barrier Reef (Babcock et al. 1986). Thus, the mature gametes of the transplanted colonies in NT disappeared between 17 August (Lunar day 22) and 4 October (Lunar day 10) (Fig. 1), indicating that spawning possibly occurred in September. This was similar to the spawning month of the native colonies in NT

In ST, the mature gametes in $56 \%$ of the transplanted colonies $(n=9)$ disappeared between 8 July (Lunar day 11) and 9 August (Lunar day 14), indicating that spawning of these colonies probably occurred in July (Fig. 1). The gradual decline in transplanted colonies containing mature gametes occurred between August and October 1995, suggesting that the release of gametes probably extended for several months or that the gametes were resorbed. On the other hand, the decline in native ST colonies containing mature gametes occurred between 6 September (Lunar day 12) and 6 October (Lunar day 12) 1995, indicating that spawning possibly began in September.

These results suggest that the transplanted colonies in NT may spawn in the same month as the original ST population and the native NT population. However, the spawning of the transplanted colonies in ST began 2 mo earlier than the original NT population and the native ST population.
Oocyte diameters of the transplanted colonies in NT were similar to those of the native NT population in June and July 1995 (Fig. 2). Oocyte diameters (mean \pm 1. SD) of the transplanted colonies in ST (84.7 \pm $20.4 \mu \mathrm{m}, \mathrm{n}=38 ; 130.6 \pm 49.0 \mu \mathrm{m}, \mathrm{n}=72$; in April 1994 and May 1995, respectively) were significantly larger than those of the original NT population $(43.9 \pm 9.2 \mu \mathrm{m}$, $\mathrm{n}=34 ; 77.5 \pm 1.2 .2 \mu \mathrm{m}, \mathrm{n}=48$; in April 1994 and May 1995, respectively) in April $1994(F=42.9, \mathrm{p}<0.001)$ and May 1995 ( $F=19.1, p<0.001)$ (Fig 2). This indicates that the early stage oocytes of the transplanted colonies in ST grew faster than those of the original colonies in NT. These data also support the previous inference that the spawning of the transplanted colonies in ST probably started 2 mo earlier than the original NT population and the native ST population.

Mature egg size: The maximum mature egg size of the transplanted and the native colonies in NT was similar, but was significantly larger than that of the transplanted and native colonies in ST (Table 1). Results of 2-way ANOVA indicated that mature egg size was influenced by both location and population (Table 1).

Discussion. This study illustrates that reproductive traits, such as reproductive timing and mature egg size, of the reef coral Echinopora lamellosa are plastic. Similar phenotypic plasticity in morphology and physiology of corals has been reported in other studies (Foster 1979, Falkowski \& Dubinsky 1981, Willis 1985, Bruno \& Edmunds 1997).

The early stage oocytes grew faster and the spawning began 2 mo earlier in the transplanted ST colonies,

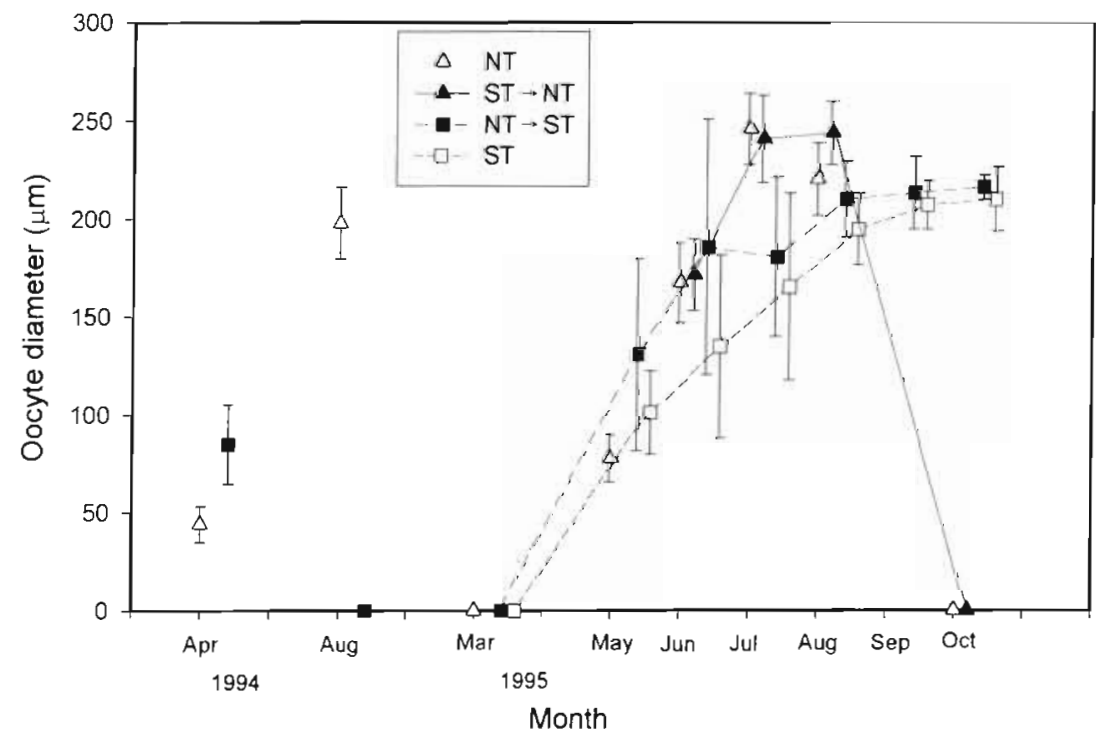

Fig. 2. Echinopora lamellosa. Variation of mean oocyte diameters of the native and transplanted colonies in northern Taiwan (NT) and southern Taiwan (ST). Error bars indicate $1 \mathrm{SD}$ 
Table 1. Mean mature egg size ( $\mu \mathrm{m}$, in diameter) and 2-way ANOVA for effects of population (Pop) and location (Loc) on mature egg diameter. NT: northern Taiwan population; ST southern Taiwan population

\begin{tabular}{|lrrrrc|}
\hline Population & Time & n & Mean & 1 SD \\
\hline NT & Jul 1995 & 72 & 236.8 & 23.9 \\
ST $\rightarrow$ NT & Jul 1995 & 72 & 236.7 & 18.8 \\
NT $\rightarrow$ ST & Sep 1995 & 72 & 213.6 & 19.3 \\
ST & Sep 1995 & 72 & 203.9 & 14.2 \\
\multicolumn{5}{c}{ Two-way ANOVA } & \\
Source & SS & df & MS & $F$ & p \\
\hline Population & 1744.9 & 1 & 1744.9 & 4.7 & $<0.05$ \\
Location & 56221.4 & 1 & 56221.4 & 149.9 & $<0.001$ \\
Pop $\times$ Loc & 1645.9 & 1 & 1645.9 & 4.4 & $<0.05$ \\
Error & 106512.8 & 284 & 375.0 & & \\
\hline
\end{tabular}

in comparison with those of the original populations in NT. This indicates that gametogenesis of corals from colder waters (NT) may be accelerated by warmer seawater temperatures. This phenomenon is similar to the differences of mass spawning of corals between inshore and offshore reefs in the Great Barrier Reef (Babcock et al. 1986). The earlier spawning on inshore reefs has been attributed to earlier rising of sea temperatures.

The difference in reproductive timing between the transplanted colonies and the native colonies in ST indicates that the ultimate selective forces molding the reproductive timing of the native $S T$ population may differ from the proximate cues inducing the gametogenesis and spawning of the transplanted colonies. Since the range of sea temperatures in ST is smaller than NT, the adequate reproductive season for coral populations in ST is longer and the transplanted colonies may have an extended reproductive season (Dai et al. 1992, Fan \& Dai 1995). However, under this circumstance, sea temperature may not act as a prime selection force controlling the timing of coral reproduction (Shlesinger \& Loya 1985, Soong 1991) and the reproductive timing of the native Echinopora lamellosa may have been molded by other factors, e.g. seasonal disturbances of typhoons and rainfall (Fan \& Dai 1995).

For those colonies transplanted from ST to NT, the timing of gametogenesis and spawning was similar to those of the native NT population. Similar phenomena were also found in the coral colonies of Echinophyllia aspera transplanted from ST to NT, although the timing of gametogenesis and spawning was different between the native populations at the 2 localities (Fan 1996). The similarities in reproductive activities of different species in response to transplantation may indicate the functional importance of reproductive timing in this habitat. Variations in physical factors such as annual sea temperature range and turbidity, which may determine the most favorable spawning period, appear to be more pronounced in NT than in ST (Dai et al. 1992, Fan \& Dai 1995). Such an environment may more sharply define the period of time during which conditions are optimal for reproductive success, thereby entraining the reproductive season of corals into a relatively brief period of time (Oliver et al. 1988). In other words, this environmental condition may act as a canalization effect that modifies the reproductive activity via direct physiological constraint over the optimal period for gamete development and spawning.

The greater egg size of populations in high latitude reefs has been found in Pocillopora verrucosa (Fadlallah 1985, Sier \& Olive 1994) and Echinopora lamellosa (Fan \& Dai 1995). This phenomenon may reflect an increasing investment in larval survivorship and represent a response to the unfavorable environment at higher latitudes (Sier \& Olive 1994). The transplanted colonies of E. lamellosa in NT produced larger mature eggs, which are the characteristics of the native NT population, and may be considered as an adaptive response to increase reproductive success.

Acknowledgements. We thank Y. C. Tsay, M. C. Lin and D. S. Chen for their assistance in the field and 3 anonymous reviewers for their valuable comments. This study was supported by a grant from the National Science Council, ROC (NSC 81-0209-B-002A-502)

\section{LITERATURE CITED}

Babcock RC, Bull GD, Harrison PL, Heyward AJ, Oliver JK, Wallace CC, Willis BL (1986) Synchronous spawnings of 105 scleractinian coral species on the Great Barrier Reef. Mar Biol 90:379-394

Babcock RC, Willis BL, Simpson CJ (1994) Mass spawning of corals on a high latitude coral reef. Coral Reefs 13:161-169

Bruno JF, Edmunds PJ (1997) Clonal variation for phenotypic plasticity in the coral Madracis mirabilis. Ecology 78 : $2177-2190$

Dai CF, Soong K, Fan TY (1992) Sexual reproduction of corals in northern and southern Taiwan. Proc 7th Int Coral Reef Symp 1:448-455

Fadlallah YH (1.985) Sexual reproduction in Pocillopora verrucosa at Yanbu, Saudi Arabia. Proc 5th Int Coral Reef Congr 4:313-318

Falkowski PG, Dubinsky Z (1981) Light-shade adaptation of Stylophora pistillata, a hermatypic coral from the Gulf of Eilat. Nature 289:172-174

Fan TY (1996) Life histories and population dynamics of foliaceous corals in northern and southern Taiwan. PhD thesis, National Taiwan University, Taipei

Fan TY, Dai CF (1995) Reproductive ecology of the scleractinjan coral Echinopora lamellosa in northern and southern Taiwan. Mar Biol 123:565-572

Fan TY, Dai CF (1998) Sexual reproduction of the scleractinian coral Merulina ampliata in southern Taiwan. Bull Mar Sci 62:897-904 
Foster AB (1979) Phenotypic plasticity in the reef corals Montastraea annularis (Ellis \& Solander) and Siderastrea siderea (Ellis \& Solander). J Exp Mar Biol Ecol 39:25-54

Harrison PL, Wallace CC (1990) Reproduction, dispersal and recruitment of scleractinian corals. Chap 7. In: Dubinsky Z (ed) Ecosystems of the world, Vol 25. Coral reefs. Elsevier, Amsterdam, p 133-207

Oliver JK, Babcock RC, Harrison PL, Willis BL (1988) Geographic extent of mass coral spawning: clues to ultimate causal factors. Proc 6th Int Coral Reef Symp 2:803-810

Richmond RH, Hunter CL (1990) Reproduction and recruitment of corals: comparisons among the Caribbean, the Tropical Pacific, and the Red Sea. Mar Ecol Prog Ser 60:185-203

Shlesinger Y, Loya Y (1985) Coral community reproductive patterns: Red Sea versus the Great Barrier Reef. Science

Editorial responsibility: Charles Birkeland (Contributing Editor), Mangilao, Guam
228:1333-1335

Sier CJS, Olive PJW (1994) Reproduction and reproductive variability in the coral Pocillopora verrucosa from the Republic of Maldives. Mar Biol 118:713-722

Soong K (1991) Sexual reproductive patterns of shallowwater reef corals in Panama. Bull Mar Sci 49:832-846

Stearns SC (1992) The evolution of life history. Oxford Unıversity Press, New York

Willis BL (1985) Phenotypic plasticity versus phenotypic stability in the reef corals Turbinaria mesenterina and Pavona cactus. Proc 5th Int Coral Reef Cong 4:107--112

Willis BL, Babcock RC, Harrison PL, Oliver JK, Wallace CC (1985) Patterns in the mass spawning of corals on the Great Barrier Reef from 1981 to 1984. Proc 5th Int Coral Reef Cong 4:343-348

Submitted: March 17, 1999, Accepted: November 3, 1999 Proofs received from author(s): November 29, 1999 